\title{
Sex, stimulus, association strength, duration and rate of repetition in semantic satiation'
}

FRED SHIMA

UNIVERSITY OF CALIFORNIA AT LOS ANGELES

Forty-eight males and 48 females repeated either the test word (experimental) or a nonsense syllable (control) under different speeds and durations for semantic satiation in a "posttest only" design, with no pre-repetition word as sociations or semantic differential ratings. No experimental satiation was noted in number of associations and frequency of first associate, and experimental generation was surprisingly found in semantic ratings.

There are two types of semantic satiation, connotative and associative (Amster, 1964). In the first case, satiation is determined by lower semantic differential ratings after repetition, and in the second case, which is of main interest here, by decreased number and lower commonality of word associations to the repeated word. Kanungo \& Lambert (1963) found no decrease in total number of associations although less relevant responses were noted, and Smith \& Raygor (1956) also reported less common associates following visual self-satiation. On the other hand, Fillenbaum (1963, Experiments I-IV) found no difference between experimental and control conditions, except in Experiment $V$ where experimental Ss gave more common associations than control after a short 4-sec. repetition treatment.

The present experiment is a parametric study designed to discover the specific conditions for satiation or even generation effects, especially possible interactions among variables commonly manipulated in semantic satiation studies (duration and rate of repetition) as well as factors rarely examined (sex and association strength of test words), and the relations between several indices of semantic satiation:

Method

Ss were 48 male and 48 female undergraduates drawn from UCLA introductory psychology courses.

Six monosyllabic nouns, the test words, were taken from the Palermo \& Jenkins (1964) word association norms for 1000 college students. The three high association words, BOY, DOG, LAMP, each evoked one association $70.4 \%, 67.9 \%$ and $70.6 \%$, respectively. Rates for the three low association words, CARS, CHEESE, MOON, were $10.7 \%, 10.6 \%$ and $21.6 \%$, respectively. The six nonsense syllables were VAY, NUR, TEB, ZEM, PAJ and KUD. The three generation scales (large-small, fastslow, wide-narrow) and three satiation scales (beautiful -ugly, active-passive, good-bad) were the same used by Yelen \& Schulz (1963, Experiment IV).

Each male and female $S$ was randomly assigned to one of eight treatments. S first was trained to match the repetition rate of an electric metronome set at either 1 or 3 beats per second. Then $S$ repeated the first of either six test words or six nonsense syllables for either 5 or $15 \mathrm{sec}$. Immediately after repetition, S wrote as many associations as possible to the test word in $1 \mathrm{~min}$., followed by rating of the test word on the six semantic differential scales. A 30-sec. interval during which the metronome was on at the appropriate rate preceded the next repetition period. So the sequence was repetition, word association, semantic rating, 30-sec. "refresher" repetition training with metronome, repetition, word association, and so on. In sum, a $2^{5}$ factorial design was used: males vs. female; experimental test word vs. control nonsense syllable repetition; fast vs. slow rate; 5 vs. 15 sec. duration; and high vs. low association test words (a repeated measure).

The stimuli to be repeated were presented on $3 \times 5$ cards, said out loud once, removed, then repeated at the given rate and duration. In each group, order of words or nonsense syllables was counterbalanced.

Number of associations was obtained by adding the number of different associations written for each test word. Frequency of the first association was checked using the Palermo \& Jenkins norms. The most common response was given a score of 1 down to 5 for the fifth most common, and 6 for any other associate. Polarity scoring of semantic scales was used, with the midpoint of the 7-point scale as 0 , and moving from 1 to 2 to 3 from the midpoint to either extreme. Semantic satiation would be reflected in smaller number of associations, less common first associates, and more neutral or lower scale ratings.

\section{Results and Discussion}

Analysis of variance was carried out for all three dependent variables. No significant differences between experimental and control in associative satiation resulted, but the experimental group displayed significantly higher semantic ratings than the control group $(p<.05)$, and this result indicated generation. High association words showed significantly lower number of associations, more common first associates, and lower semantic ratings. Females gave significantly higher semantic ratings than males. Fast repetition evoked significantly greater number of associations than slow. None of the interactions were significant, except for one three-way and one four-way interaction in number of associations, both uninterpretable.

Schulz, Weaver, \& Radtke (1965) reported no connotative satiation when a "posttest only" design, the same 
used here, was presented, and the data here showed no evidence of associative satiation either. Perhaps the experimental effect was washed out by combining the 5-sec. duration (generation) and 15-sec. (satiation) conditions. Such a possibility might be noted in a Treatment by Duration interaction, but none was found across all three satiation indices. Another question may involve the intervention of the word association period between repetition and ratings, which could have dissipated any initial satiation. This might explain experimental and control groups giving similar ratings, but certainly not the control group showing significantly lower scores as was found. The result is consistent with Schulz et al's trend for lower polarity in their control groups.

\section{References}

Amster, H. Semantic satiation and generation: Learning? Adaptation? Psychol. Bull., 1964, 62, 273-286.

Fillenbaum, $\mathbf{S}$. Verbal satiation and changes in meaning of related items. J. verbal Learn. verbal Behav., 1963, 2, 263-271.

Kanungo, R. N., \& Lambert, W. E. Semantic satiation and meaningfulness. Amer. J. Psychol., 1963, 76, 421-428.

Palermo, D. S., \& Jenkins, J. J. Word association norms. Minneapolis: University of Minnesota Press, 1964.

Schulz, R. W., Weaver, G. E., \& Radtke, R. C. Verbal satiation?? Psychon. Sci., 1965, 2, 43-44.

Smith, D. E., \& Raygor, A. L. Verbal satiation and personality. J. abnorm. soc. Psychol., 1956, 52, 323-326.

Yelen, D. R., \& Schulz, R. W. Verbal satiation? J, verbal Learn. verbal Behav., 1963, 1, 372-377.

\section{Note}

1. The help from Drs. J. P. Das and Charles Nakamura is gratefully acknowledged. 